\title{
On the Diameter of Certain Riemannian Manifolds
}

\author{
By \\ Kunio Sugahara
}

\section{§. Introduction}

The purpose of this paper is to estimate the lower bound for the diameters of certain Riemannian manifolds.

Let $M$ be a compact connected $C^{\infty}$ Riemannian manifold and $K$ its sectional curvature. Let $p$ be a point of $M$ and $G_{p}$ the group of all isometries of $M$ which fix the point $p$. We denote by $d(M)$ the diameter of $M$.

We shall prove:

Theorem A. Assume $K \leqq 1$ and there exists a point $p$ in $M$ such that $\operatorname{dim} G_{p} \geqq 1$. Then $d(M) \geqq \pi / 2$. If the equality $d(M)=\pi / 2$ holds, then $K \equiv 1$. Therefore, in case of even dimension, the equality holds if and only if $M$ is isometric to the real projective space of constant curvature 1.

From this theorem we obtain an alternative proof of the following

Corollary (cf. [2]). Assume $K \leqq 0$. Then $\operatorname{dim} G_{p}=0$ for any point p of $M$.

We shall also prove:

Theorem B. Assume $0<K \leqq 1$ and $M$ is of even dimension, then $d(M) \geqq \pi / 2$. The equality $d(M)=\pi / 2$ holds if and only if $M$ is isometric to the real projective space of constant curvature 1 .

Received September 6, 1975. 
Remark 1.1. The assumption $K \leqq 1$ is not essential. Since $M$ is compact, in order to fulfill the assumption, we have only to change the metric by a constant factor.

Remark 1.2. In Theorem B, the assumption that $M$ is of even dimension cannot be eliminated. In fact, even if $M$ is of constant curvature $1, d(M)$ can be less than $\pi / 2$ (cf. [8]).

Next we shall study the relation between the cut locus and the conjugate locus. And we shall prove:

Theorem $\mathbb{A}^{\prime}$ 。. Assume $K \leqq 1$ and there exists a point $p$ in $M$ such that $\operatorname{dim} G_{p} \geqq 1$. Further assume that $M$ is simply connected and that $\operatorname{dim} M$ is 3 or 4 . Then $d(M) \geqq \pi$ 。

Corresponding to Theorem B, the following theorem is known.

Theorem $\mathbf{B}^{\prime}$. Assume $0<K \leqq 1$ and $M$ is simply connected and of even dimension. Then $d(M) \geqq \pi$.

Remark 1.3. If $M$ is of dimension 2, we need less assumption. In fact, we have: If $M$ is of dimension 2 and simply connected and if $K \leqq 1$, then $d(M) \geqq \pi$ (cf. Theorem 6.1).

Note: In this paper, we assume that the manifold $M$ is always compact and $\operatorname{dim} M \geqq 2$.

\section{§2. Examples}

The complex $n$-space $\mathbb{C}^{n}(n \geqq 2)$ is considered to be the Euclidean $2 n$-space by the usual hermitian inner product. Let $S^{2 n-1}$ be the unit sphere in $\mathbb{C}^{n}$. Then $S^{2 n-1}$ is of constant curvature 1 , concerning to the induced metric by the inclusion $S^{2 n-1} \subset \mathbb{C}^{n}$. And the unitary transformations $U(n)$ act on $S^{2 n-1}$ as isometries.

(1) Lens spaces. Let $m$ be an integer greater than 1 and let $r_{1}, \ldots$, $r_{n}$ be primitive $m$-th roots of 1 (not necessarily distinct). We define a unitary matrix $U$ by

$$
\left(\begin{array}{lll}
r_{1} & & 0 \\
& \ddots & \\
0 & & r_{n}
\end{array}\right) .
$$


Let $\Gamma$ be the subgroup of $U(n)$ generated by $U$. Then $\Gamma$ is a finite group which acts freely on $S^{2 n-1}$. Hence $M=S^{2 n-1} / \Gamma$ is a manifold which inherits the Riemannian structure of constant curvature $1 . M$ is called a lens space. Let $G$ be the subgroup of $U(n)$ consisting of matrices

$$
\left(\begin{array}{lll}
1 & & 0 \\
& \lambda & \\
& & \ddots \\
0 & & \lambda
\end{array}\right)
$$

such that $\lambda \in \mathbb{C}$ and $|\lambda|=1$ and let $p$ be a point of $M$ which is represented by the vector $(1,0, \ldots, 0) \in \mathbb{C}^{n}$. Since $G$ is contained in the normalizer of $\Gamma$, each element of $G$ is considered to be an isometry of $M$ and fixes the point $p$. Therefore we obtain $\operatorname{dim} G_{p} \geqq \operatorname{dim} G=1$. As for the lens spaces, $d(M)=\pi / 2$ (cf. [8]).

Remark 2.1. If $M$ is of constant curvature 1 with $d(M)=\pi / 2$ and if $\operatorname{dim} M$ is 3 or 5 , then the fundamental group is cyclic (cf. [8]). Hence $M$ is a lens space.

(2) Let $\Gamma$ be a subgroup of $U(4)$ generated by the following matrices

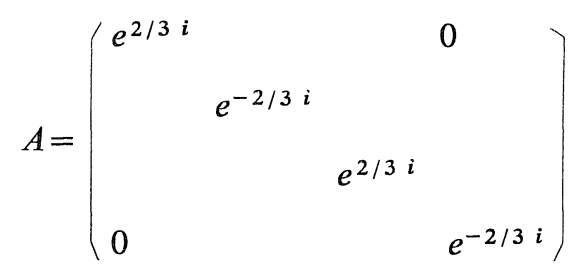

and

$$
B=\left(\begin{array}{rrrr}
0 & -1 & 0 & 0 \\
1 & 0 & 0 & 0 \\
0 & 0 & 0 & -1 \\
0 & 0 & 1 & 0
\end{array}\right)
$$

Then $\Gamma$ is a non-commutative finite group which acts freely on $S^{7}$. Hence $M=S^{7} / \Gamma$ is also a Riemannian manifold of constant curvature 1 . we put 


$$
G=\left\{\left(\begin{array}{ccc}
1 & & 0 \\
& 1 & \\
& \lambda & \\
0 & & \lambda
\end{array}\right) ; \lambda \in \mathbb{C} \text { and }|\lambda|=1\right\}
$$

Then each element of $G$ acts on $M$ as an isometry and fixes the point $p$ which is represented by the vector $(1,0,0,0) \in \mathbb{C}^{4}$. Hence $\operatorname{dim} G_{p}$ $\geqq \operatorname{dim} G=1$. In this case, we also have $d(M)=\pi / 2$ (cf. [8]). However $M$ is not a lens space since $\Gamma$ is not cyclic.

\section{§3. Preliminaries}

We denote by $<,>$ the Riemannian metric of $M$. We denote by $\dot{c}(t)$ the velocity vector of a curve $c:[a, b] \rightarrow M$ at $c(t)$ and by $L(c)$ the length of $c$. We denote by $d(p, q)$ the distance between two points $p$ and $q$ of $M$. We denote by $T_{p} M$ the tangent space to $M$ at $p$ and by $\exp _{p}$ the exponential mapping of $T_{p} M$ to $M$. We put $B_{p}(r)=\left\{v \in T_{p} M\right.$; $\|v\|<r\}$ and $S_{p}(r)=\left\{v \in T_{p} M ;\|v\|=r\right\}$. Then, by the theorem of MorseSchoenberg, we know that if $K \leqq 1$, then the map $\left.\exp _{p}\right|_{B_{p}(\pi)}$ is of maximal rank at any point $p$ of $M$. The following proposition is known (cf. [1] p. 149, Theorem 2).

Proposition 3.1. Assume that the map $\left.\exp _{p}\right|_{B_{p}(r)}$ is of maximal rank. Let $\gamma:[a, b] \rightarrow B_{p}(r)$ be a piecewise $C^{\infty}$ curve such that $\gamma(a)=0$. Then we have $L\left(\exp _{p} \circ \gamma\right) \geqq\|\gamma(b)\|$. The equality $L\left(\exp _{p} \circ \gamma\right)=\|\gamma(b)\|$ holds if and only if $\exp _{p} \circ \gamma$ is a geodesic with an appropriate change of parametrization.

Making use of this proposition, we obtain

Lemma 3.2. Assume that the map $\left.\exp _{p}\right|_{B_{p}(r)}$ is of maximal rank. Let $c:[a, b] \rightarrow M$ be a piecewise $C^{\infty}$ curve emanating from $p$. Assume that the following (i) or (ii) holds.

(i) $L(c)<r$.

(ii) $L(c) \leqq r$ and $c$ is not a geodesic with any reparametrization. Then there exists a unique piecewise $C^{\infty}$ curve $\gamma:[a, b] \rightarrow B_{p}(r)$ such that $\gamma(a)=0$ and $\exp _{p} \circ \gamma=c$. 
$\gamma$ is called the lift of $c$ to $T_{p} M$.

Proof. Since the map $\left.\exp _{p}\right|_{B_{p}(r)}$ is of maximal rank, $\gamma$ is unique if it exists. By our assumption, there is a number $s(a<s \leqq b)$ such that there is the lift $\gamma_{s}$ of $\left.c\right|_{[a, s]}$ to $T_{p} M$. Let $s_{0}$ be the least upper bound of such $s$. We note that, for any $s$ and $s^{\prime}\left(s<s^{\prime}\right), \gamma_{s}=\gamma_{s^{\prime}}$ on $[a, s]$. Therefore the curve $\gamma:\left[a, s_{0}\right) \rightarrow B_{p}(r)$ can be defined by $\gamma(t)=\gamma_{s}(t)$ for $a \leqq t \leqq s<s_{0}$. By Proposition 3.1 and our assumption, we easily see that $\gamma(s)\left(s \rightarrow s_{0}\right)$ converges to a point of $B_{p}(r)$. Hence it follows that there is the lift $\gamma_{s_{0}}$ of $\left.c\right|_{\left[a, s_{0}\right]}$ to $T_{p} M$. So, if $s_{0}<b$, the lift $\gamma_{s_{0}}$ can be extended, which is contradictory to the definition of $s_{0}$. Therefore $s_{0}=b$ and $\gamma_{s_{0}}$ is the lift of $c$ to $T_{p} M$.

Q.E.D.

Lemma 3.3. Assume $K \leqq 1$. Let $v$ and $w$ be tangent vectors contained in $B_{p}(\pi)$ such that $\langle v, w\rangle=0$. Let $\gamma:[a, b] \rightarrow B_{p}(\pi)$ be a piecewise $C^{\infty}$ curve connecting $v$ and $w$. Then $L\left(\exp _{p} \circ \gamma\right) \geqq\|v\|$ if $\|v\| \leqq \pi / 2$.

Proof. Let $S^{n}(n=\operatorname{dim} M)$ denote the $n$-sphere of constant curvature 1. Let $p_{0}$ be a point of $S^{n}$ and $I: T_{p} M \rightarrow T_{p_{0}} S^{n}$ a linear isometry. Then, by Rauch's comparison theorem (cf. [1], p. 250, Theorem 14), we have

$$
\left\|\widetilde{\left(\exp _{p^{\circ}} \gamma\right)}(t)\right\| \geqq\left\|\widehat{\left(\exp _{p_{0}} \circ I \circ \gamma\right)}(t)\right\| \quad(a \leqq t \leqq b) .
$$

Hence it follows that

$$
L\left(\exp _{p} \circ \gamma\right) \geqq L\left(\exp _{p_{0}} \circ I \circ \gamma\right) .
$$

On the other hand, it is clear that

(3) If $\|I v\| \leqq \pi / 2$,

$$
d\left(\exp _{p_{0}} \circ I v, \exp _{p_{0}} \circ I w\right) \geqq d\left(\exp _{p_{0}} \circ I v, p_{0}\right)=\|I v\| .
$$

The lemma follows from (2) and (3).

Q.E.D.

Remark 3.4. In the proof above, if we further assume that $\|v\|$ $=L\left(\exp _{p} \circ \gamma\right)=\pi / 2$, then it follows from (2) and (3) that

$$
L\left(\exp _{p} \circ \gamma\right)=L\left(\exp _{p_{0}} \circ I \circ \gamma\right)=d\left(\exp _{p_{0}} \circ I v, \exp _{p_{0}} \circ I w\right) .
$$


(4) combined with (1) yields

$$
\left\|\widehat{\left(\exp _{p} \circ \gamma\right)}(t)\right\|=\left\|\widehat{\left(\exp _{p_{0}} \circ I \circ \gamma\right)}(t)\right\| \quad(a \leqq t \leqq b) .
$$

Hence we see from (4) that $\exp _{p_{0}} \circ I_{\circ} \gamma$ is a shortest geodesic with an appropriate change of parametrization. And if $\exp _{p} \circ \gamma$ is a geodesic with the original parametrization, so is $\exp _{p_{0}} \circ I \circ \gamma$.

Lemma 3.5. Assume $K \leqq 1$. Let $\alpha: \mathbb{R} \rightarrow S_{q}(\pi / 2)$ be a curve such that $a=\exp _{q} \circ \alpha$ is a geodesic with $\|\dot{a}(t)\|=1(t \in \mathbb{R})$. We put $\exp _{q} \alpha(0)=p$. Let $v \in T_{p} M$ be the initial tangent of the geodesic $\exp _{q}(1-t) \alpha(0)(0 \leqq t \leqq 1)$. Then $\exp _{p}\{\lambda \dot{a}(0)+\mu v ; \lambda, \mu \in \mathbb{R},\|\lambda \dot{a}(0)+\mu v\|=\pi$ and $\mu \geqq 0\}$ is a single point.

Proof. First we note that $\langle\dot{a}(0), v\rangle=0$. Let $\left(\varepsilon_{1}, \varepsilon_{2}\right)$ be the connected component of $\{t \in \mathbb{R} ; \alpha(t) \neq-\alpha(0)\}$ which contains 0 . We define a family of curves $c_{t}:[0,1] \rightarrow M\left(\varepsilon_{1}<t<\varepsilon_{2}\right)$ by

$$
c_{t}(s)= \begin{cases}\exp _{p} 2 s v & (0 \leqq s \leqq 1 / 2) \\ \exp _{q}(2 s-1) \alpha(t) & (1 / 2 \leqq s \leqq 1)\end{cases}
$$

Then, by Lemma 3.2, there are lifts $\gamma_{t}$ of $c_{t}$ to $T_{p} M$. Let $S^{n}(n=\operatorname{dim} M)$ be the $n$-sphere of constant curvature 1. Let $p_{0}$ be a point of $S^{n}$ and $I: T_{p} M \rightarrow T_{p_{0}} S^{n}$ a linear isometry. We define $b_{t}:[1 / 2,1] \rightarrow S^{n}$ by $b_{t}$ $=\exp _{p_{0}} \circ I_{\circ} \gamma_{t[1 / 2,1]}$. Then by the definition of $c_{t}$ and $b_{t}$, it is clear that

$$
\dot{b}_{t}(1 / 2) \neq-\dot{b}_{0}(1 / 2) \quad\left(\varepsilon_{1}<t<\varepsilon_{2}\right) \text {. }
$$

On the other hand, we have

$$
L\left(\left.\exp _{p} \circ \gamma_{t}\right|_{[1 / 2,1]}\right)=\left\|\gamma_{t}(1 / 2)\right\|=\pi / 2
$$

Hence it is easily seen from Remark 3.4 that:

(7) $\quad b_{t}$ is the shortest geodesic connecting $\exp _{p_{0}} \circ I v$ and $\exp _{p_{0}} \circ I(t \dot{a}(0))$;

$$
\left\|\dot{b}_{t}(s)\right\|=\pi\left(\varepsilon_{1}<t<\varepsilon_{2} \text { and } 1 / 2 \leqq s \leqq 1\right)
$$

(9) The angle between two initial vectors $\dot{b}_{t}(1 / 2)$ and $\dot{b}_{0}(1 / 2)$ is equal to $\|t \dot{a}(0)\|=|t|$. 
By the definition of $\varepsilon_{1}$ and $\varepsilon_{2}$ and by (8), we have

$$
\lim _{t \rightarrow \varepsilon_{1}+0} \dot{b}_{t}(1 / 2)=-\dot{b}_{0}(1 / 2)=\lim _{t \rightarrow \varepsilon_{2}-0} \dot{b}_{t}(1 / 2) .
$$

It follows from (6), (9) and (10) that $-\varepsilon_{1}=\varepsilon_{2}=\pi$. We fix a vector $\xi_{\lambda}=\lambda \dot{a}(0)+\mu v\left(\mu \geqq 0\right.$ and $\left.\left\|\xi_{\lambda}\right\|=\pi\right)$ and first study the case that $\lambda>0$. We obtain from (7) that the half line $\mathbb{R}_{+} I \xi_{\lambda}\left(\mathbb{R}_{+}=\{r \in \mathbb{R} ; r \geqq 0\}\right)$ and $I \circ$ $\left.\gamma_{t}\right|_{[1 / 2,1]}$ have a single intersection which we denote by $I \circ \gamma_{t}\left(s_{t}\right)$ where $1 / 2<s_{t} \leqq 1$. Then it is clear from (9) and (10) that

$$
\lim _{t \rightarrow \pi-0} s_{t}=1 \text { and } \lim _{t \rightarrow \pi-0}\left\|I \circ \gamma_{t}\left(s_{t}\right)\right\|=\pi \text {. }
$$

Hence we have

$$
\begin{aligned}
& d\left(\exp _{p} \xi_{\lambda}, \exp _{p} \pi \dot{a}(0)\right)=\lim _{t \rightarrow \pi-0} d\left(\exp _{p} \circ \gamma_{t}\left(s_{t}\right), \exp _{p} t \dot{a}(0)\right) \\
& \leqq \lim _{t \rightarrow \pi-0} L\left(\left.\exp _{p} \circ \gamma_{t}\right|_{\left[s_{t}, 1\right]}\right)=\lim _{t \rightarrow \pi-0}\left(1-s_{t}\right) \pi=0
\end{aligned}
$$

In case that $\lambda<0$, by the similar argument we see that

$$
\exp _{p} \ddot{\xi}_{\lambda}=\exp _{p}(-\pi \dot{a}(0))
$$

By the continuity of the map $\exp _{p}$, we obtain

$$
\lim _{\lambda \rightarrow-0} \exp _{p} \xi_{\lambda}=\exp _{p} \xi_{0}=\lim _{\lambda \rightarrow+0} \exp _{p} \xi_{\lambda}
$$

proving the lemma.

Q.E.D.

We define the cut locus $C(p)$ of $p$ in $T_{p} M$ by

$$
C(p)=\left\{v \in T_{p} M ; \begin{array}{ll}
d\left(p, \exp _{p} t v\right)=\|t v\| & (0 \leqq t \leqq 1) \\
d\left(p, \exp _{p} t v\right)<\|t v\| & (1<t)
\end{array}\right\}
$$

and the set $\exp _{p} C(p)$ is also called the cut locus of $p$ and denoted by $C(p)$.

The following theorems are known.

Theorem 3.6 (cf. [7]). Assume $K \leqq 1$ and there is a point $p$ in $M$ such that $C(p)=S_{p}(\pi / 2)$. Then $M$ is isometric to the real projective space of constant curvature 1. 
Theorem 3.7 (cf. [6]). Assume $K \leqq 1$ and there is a point $p$ in $M$ such that $C(p)=S_{p}(\pi)$ and $\exp _{p} C(p)$ is a single point. Then $M$ is isometric to the sphere of constant curvature 1.

In this paper, we use a weaker version of Theorem 3.7:

Theorem 3.8. Assume $K \leqq 1$ and there is a point $p$ in $M$ such that $\exp _{p} S_{p}(\pi)$ is a single point. Then $K \equiv 1$.

This theorem can be proved by the similar argument to that of section 2 in [6].

\section{§4. Fixed Points of Isometries}

In this section, $p$ denotes an arbitrary, but fixed point of $M$ and we assume that $\operatorname{dim} G_{p} \geqq 1$ and $K \leqq 1 . G_{p}$ is sometimes considered to be a subgroup of the linear orthogonal transformations $O\left(T_{p} M\right)$ by the linear isotropy representation. Let $G$ denote the identity component of $G_{p}$ and let $F$ be the set of points of $M$ which are fixed by $G$.

We note that $F$ is a finite union of totally geodesic closed submanifolds of $M$.

Lemma 4.1. Let $x$ and $y$ be two points of $F$ and $c:[0,1] \rightarrow M$ a geodesic connecting $x$ and $y$ such that $L(c)<\pi$. Then $c$ is contained in $F$.

Proof. Since $\exp _{x} \dot{c}(0)=y \in F, \exp _{x} \dot{c}(0)=G\left(\exp _{x} \dot{c}(0)\right)=\exp _{x} G \dot{c}(0)$. Hence it follows from the theorem of Morse-Schoenberg with $\|\dot{c}(0)\|=\mathbb{L}(c)<\pi$ that $\dot{c}(0)$ is fixed by $G$, which implies that $\dot{c}(0)$ is tangent to $F$. Since $F$ is totally geodesic, $c$ is contained in $F$.

Q.E.D.

Proposition 4.2. Let $q$ be a point of $F$ and $v$ a tangent vector to $M$ at $q$ such that $\|v\| \leqq \pi / 2$ and $\left.<v, T_{q} F\right\rangle=0$. Then $d\left(F, \exp _{q} v\right)=\|v\|$. Therefore the inequality $\|w\| \geqq \pi / 2$ holds for any $w \in C(q) \cap\left(T_{q} F\right)^{\perp}$.

Proof. Suppose that $d\left(F, \exp _{q} v\right)<\|v\|$. Then there is a point $q^{\prime} \in F$ such that $d\left(q^{\prime}, \exp _{q} v\right)=d\left(F, \exp _{q} v\right)$. Let $a:[0,1] \rightarrow M$ be a shortest geodesic from $\exp _{q} v$ to $q^{\prime}$. We define a curve $c:[0,1] \rightarrow M$ by 


$$
c(t)= \begin{cases}\exp _{q} 2 t v & (0 \leqq t \leqq 1 / 2) \\ a(2 t-1) & (1 / 2 \leqq t \leqq 1)\end{cases}
$$

Then we obtain $L(c)<\pi$. Hence by Lemma 3.2, there is the lift $\gamma$ of $c$ to $T_{q} M$. Since $\|\gamma(1)\|<\pi$ and $\exp _{q} \gamma(1) \in F$, it follows from Lemma 4.1 that the geodesic $\exp _{q} s \gamma(1)(0 \leqq s \leqq 1)$ is contained in $F$, i.e., $\gamma(1) \in T_{q} F$. Applying Lemma 3.3, we see $L(a)=L\left(\left.\exp _{q} \gamma\right|_{[1 / 2,1]}\right) \geqq\|v\|$, which contradicts our hypothesis.

Q.E.D.

Corollary 4.3. Let $q$ be a point of $F$. If $d(M)=\pi / 2$, then $C(q)$ $\cap\left(T_{q} F\right)^{\perp}=S_{q}(\pi / 2) \cap\left(T_{q} F\right)^{\perp}$.

We put $S_{q}^{F}=S_{q}(\pi / 2) \cap\left(T_{q} F\right)^{\perp}$.

Lemma 4.4. If $d(M)=\pi / 2$, then $\exp _{p}\left(S_{p}^{F}\right)=\exp _{q}\left(S_{q}^{F}\right)$ for any point $q$ of $F$.

Proof. Let $x$ be a point of $\exp _{q} S_{q}^{F}$. Then, by our assumption $d(M)$ $=\pi / 2$ and by Proposition 4.2, we get $d(F, x)=d(p, x)=\pi / 2$. Hence the shortest geodesic from $x$ to $p$ is normal to $F$ at $p$ and its length is $\pi / 2$. It means that $x$ is contained in $\exp _{p} S_{p}^{F}$. So we have $\exp _{q} S_{q}^{F}$ $\subset \exp _{p} S_{p}^{F}$. In the same way, we get $\exp _{q} S_{q}^{F} \supset \exp _{p} S_{p}^{F}$ Q.E.D.

Lemma 4.5. Assume $d(M)=\pi / 2$. Let $a: \mathbb{R} \rightarrow F$ be a geodesic such that $a(0)=p$. Let $v$ be a vector in $S_{p}^{F}$ and put $q=\exp _{p} v$. Then there is a curve $\alpha: \mathbb{R} \rightarrow S_{q}(\pi / 2)$ such that $\exp _{q} \circ \alpha=a$.

Proof. We define a sphere bundle $S^{F}$ over $F$ by $S^{F}=\underset{x \in F}{\cup} S_{x}^{F}$, with the projection $\pi$ induced by the projection of the tangent bundle $T M$ of $M$. We define a map $\varphi: S^{F} \rightarrow M$ by $\left.\varphi\right|_{S_{x}^{F}}=\exp _{x} \mid S_{x}^{F}$. Since the map $\left.\varphi\right|_{S_{x}^{F}}$ is of maximal rank, Lemma 4.4 implies that the subset $\varphi^{-1}(q)$ $=\left\{w \in S^{F} ; \varphi(w)=q\right\}$ of $S^{F}$ is a submanifold and $\pi: \varphi^{-1}(q) \rightarrow F$ is a covering. Hence there is a curve $\alpha_{1}: \mathbb{R} \rightarrow \varphi^{-1}(q)$ such that $\pi \circ \alpha_{1}=a$. It is clear that the curve $\alpha: \mathbb{R} \rightarrow S_{q}(\pi / 2)$ defined by the equation

$$
\exp _{q} t \alpha(s)=\exp _{a(s)}(1-t) \alpha_{1}(s) \quad(0 \leqq t \leqq 1 \quad \text { and } \quad s \in \mathbb{R})
$$

has the required property.

Q.E.D. 
Proof of Theorem $A$. The inequality $d(M) \geqq \pi / 2$ follows from Proposition 4.2. Hence we assume $d(M)=\pi / 2$ and derive $K \equiv 1$.

Case 1. The case where $\operatorname{dim} F=0$. By Corollary 4.3, we have $C(p)$ $=S_{p}(\pi / 2)$. So the assertion follows from Theorem 3.6.

Case 2. The case where $\operatorname{dim} F \geqq 1$. Let $v$ be a tangent vector to $M$ at $p$ with $<v, T_{p} F>=0$ and $\|v\|=\pi$. Let $w$ be a tangent vector to $F$ at $p$ with $\|w\|=\pi$. And let $a: \mathbb{R} \rightarrow F$ be a geodesic to which $w$ is tangent at $a(0)=p$. Then Lemma 3.5, combined with Lemma 4.5, implies that

$$
\left\{\exp _{p} v\right\}=\left\{\exp _{p}(\cos \theta w+\sin \theta v) ; 0 \leqq \theta \leqq \pi\right\}=\left\{\exp _{p} w\right\}
$$

Since any tangent vector $u$ to $M$ at $p$ with $\|u\|=\pi$ can be written in the form $\cos \theta w+\sin \theta v(0 \leqq \theta \leqq \pi)$, we obtain that $\exp _{p} S_{p}(\pi)$ is a single point. Hence the assertion follows from Theorem 3.8 .

Q.E.D.

Proof of Corollary to Theorem $A$. Suppose that both $K \leqq 0$ and $\operatorname{dim} G_{p} \geqq 1$ are satisfied. We consider the metric $r<,>(r>0)$ and denote by $d_{r}(M)$ the diameter of $M$ concerning to the metric. Then, by Theorem $\mathrm{A}$, we have $d_{r}(M) \geqq \pi / 2$. On the other hand, it is clear that $\lim _{r \rightarrow 0} d_{r}(M)=0$, which is a contradiction.

Q.E.D.

\section{§5. Riemannian Manifolds of Even-Dimension and Positive Curvature}

In this section, $M$ denotes a Riemannian manifold of even dimension with $0<K \leqq 1$.

The following theorems are known.

Theorem 5.1 (cf. [3]). If $M$ is simply connected, then $d(p, C(p)) \geqq \pi$ for any point $p$ of $M$.

Theorem 5.2 (cf. [10]). If $M$ is orientable, then it is simply connected.

By virtue of the theorems above, we obtain

Theorem 5.3. $d(p, C(p)) \geqq \pi / 2$ for any point $p$ of $M$. 
Proof. According to Theorem 5.1, we may assume that $M$ is not simply connected. Then Theorem 5.2 implies that the fundamental group of $M$ is $\mathbb{Z}_{2}$. Suppose that there is a vector $v \in C(p)\left(\subset T_{p} M\right)$ such that $\|v\|<\pi / 2$. Then there is a vector $w(w \neq v)$ in $C(p)$ such that $\exp _{p} w=$ $\exp _{1} v$. Let $\pi: \tilde{M} \rightarrow M$ be the Riemannian universal covering and put $\pi^{-1}(p)=\left\{p_{1}, p_{2}\right\}$. We define a curve $c:[0,1] \rightarrow \tilde{M}$ emanating from $p_{1}$ by

$$
\pi \circ c(t)= \begin{cases}\exp _{p} 2 t v & (0 \leqq t \leqq 1 / 2) \\ \exp _{p}(2-2 t) w & (1 / 2 \leqq t \leqq 1)\end{cases}
$$

Then it is clear by Theorem 5.1 that $c(1)=p_{2}$, which gives $d\left(p_{1}, p_{2}\right)$ $\leqq\|v\|+\|w\|<\pi$. Let $c_{1}:[0,1] \rightarrow \tilde{M}$ be a shortest geodesic from $p_{1}$ to $p_{2}$. We define another geodesic $c_{2}$ emanating from $p_{1}$ by

$$
\pi \circ c_{2}(t)=\pi \circ c_{1}(1-t) \quad(0 \leqq t \leqq 1) .
$$

Then clearly $c_{1}$ and $c_{2}$ are distinct geodesics from $p_{1}$ to $p_{2}$ with $L\left(c_{1}\right)$ $=L\left(c_{2}\right)=d\left(p_{1}, p_{2}\right)$. Hence it follows that $d\left(p_{1}, C\left(p_{1}\right)\right) \leqq d\left(p_{1}, p_{2}\right)<\pi$, which is contradictory to Theorem 5.1.

Q.E.D.

Proof of Theorem B. By Theorem 5.3, we get the inequalities $d(M)$ $\geqq d(p, C(p)) \geqq \pi / 2$ for any point $p$ of $M$. Hence the theorem follows from Theorem 3.6.

Q.E.D.

\section{§6. The Relations between the Cut Locus and the Conjugate Locus}

Let $p$ be a point of $M$. The conjugate locus $Q(p)$ of $p$ in $T_{p} M$ is defined to be the set of tangent vectors where the exponential mapping $\exp _{p}: T_{p} M \rightarrow M$ is not of maximal rank.

In case of dimension 2, the following theorem is known.

Theorem 6.1 (cf. [5], [9]). Let $M$ be a simply connected 2-dimensional Riemannian manifold. Then $C(p)$ and $Q(p)$ have an intersection for any point $p$ of $M$.

However, in case of $\operatorname{dim} M \geqq 3$, the assertion in this theorem is false 
(cf. [11]). Hence we need some further hypothesis.

Theorem 6.2 (cf. [9]). Let $M$ be a simply connected 3-dimensional Riemannian manifold. Assume that there is a point $p$ in $M$ such that $\operatorname{dim} G_{p} \geqq 1$. Then $C(p)$ and $Q(p)$ have an intersection.

Theorem 6.3。 Let $M$ be a 4-dimensional Riemannian manifold. Assume that there is a point $p$ in $M$ such that $\operatorname{dim} G_{p} \geqq 1$ and $C(p)$ $\cap Q(p)=\varnothing$. Then the Euler-Poincaré characteristic $\chi(M)$ of $M$ is less than 2.

Proof. Let $G$ be a one-parameter subgroup of $G_{p}$. Let $F$ be the set of points of $M$ which are fixed by $G$. Then $F$ is a finite union of totally geodesic closed submanifolds of $M$ with even codimensions and $\chi(F)=\chi(M)$ (cf. [4]). Let $x$ be a point of $F$. Let $c:[0,1] \rightarrow M$ be the shortest geodesic from $p$ to $x$. Since $C(p) \cap Q(p)=\varnothing$ and $x=G \circ \exp _{p} \dot{c}(0)$ $=\exp _{p} \circ G \circ \dot{c}(0), c$ is contained in $F$. Hence $F$ is connected.

Case 1. The case where $\operatorname{dim} F=0$. In this case, we have $F=\{p\}$. Hence it follows that $\chi(M)=\chi(F)=1$.

Case 2. The case where $\operatorname{dim} F=2$. We consider $F$ a Riemannian manifold with the metric induced by the inclusion $F \subset M$. And we define the cut locus and the conjugate locus of $p$ in $T_{p} F$ which we denote by $C_{F}(p)$ and $Q_{F}(p)$ respectively. As in the beginning of this proof, every shortest geodesic from $p$ to a point $F$ is contained in $F$. Hence it follows that $C_{F}(p)=C(p) \cap T_{p} F$. Since $Q(p) \supset Q_{F}(p)$, we get $C_{F}(p) \cap Q_{F}(p)=\varnothing$. So we can apply Theorem 6.1 and obtain that $F$ is not simply connected. Then it is clear that $\chi(M)=\chi(F) \leqq 1$. Q.E.D.

Corollary 6.4. Let $M$ be a simply connected 4-dimensional Riemannian manifold. Assume that there is a point $p$ in $M$ such that $\operatorname{dim} G_{p}$ $\geqq 1$. Then $C(p) \cap Q(p) \neq \varnothing$.

Proof. We put $b_{i}=\operatorname{dim} H_{i}(M ; R)$. Since $M$ is connected and simply connected, $b_{0}=b_{4}=1$ and $b_{1}=0$. By the Poincaré duality, we see $b_{3}=b_{1}$. After all we have

$$
\chi(M)=b_{0}-b_{1}+b_{2}-b_{3}+b_{4}=2+b_{2} \geqq 2 \text {. }
$$


Hence the assertion is clear.

Q.E.D.

As to the manifolds of positive curvature and even dimension, we know

Theorem 6.5 (cf. [3]). Let $M$ be a simply connected even dimensional Riemannian manifold with strictly positive curvature. Then there is a point $p$ in $M$ such that $C(p) \cap Q(p) \neq \emptyset$.

Proof of Theorem $A^{\prime}$ and Theorem $B^{\prime}$. By the theorem of MorseSchoenberg, $Q(p) \cap B_{p}(\pi)=\varnothing$ if $K \leqq 1$. Hence our assertion $d(M) \geqq \pi$ follows from Corollary 6.4 and Theorem 6.5. $\quad$ Q.E.D.

\section{References}

[1] Bishop, R. L. and Crittenden, R. J., Geometry of Manifolds, Academic Press, New York, 1964.

[2] Bochner, S., Vector fields and Ricci curvature, Bull. Amer. Math. Soc., 52 (1946), 776-797.

[3] Klingenberg, W., Contributions to Riemannian geometry in the large, Ann. of Math., 69 (1959), 654-666.

[4] Kobayashi, S., Fixed points of isometries, Nagoya Math. J., 13 (1958), 63-68.

[5] Myers, S. B., Connection between differential geometry and topology, Duke Math. J., 1 (1935), 376-391.

[6] Nakagawa, H., Riemannian manifolds with many geodesic loops, J. Math. Soc. Japan, 20 (1968), 648-654.

[7] Nakagawa, H. and Shiohama, K., On Riemannian manifolds with certain cut loci, Tôhoku Math. J., 22 (1970), 14-23.

[8] Sakai, T. and Shiohama, K., On the structure of positively curved manifolds with certain diameter, Math. Z., 127 (1972), 75-82.

[9] Sugahara, K., On the cut locus and the topology of Riemannian manifolds, J. Math. Kyoto Univ., 14 (1974), 391-411.

[10] Synge, J. L., On the connectivity of spaces of positive curvature, Quart. J. Math. (Oxford Ser.), 7 (1936), 316-320.

[11] Weinstein, A. D., The cut locus and conjugate locus of a Riemannian manifold, Ann. of Math., 87 (1968), 29-41. 
